\title{
Adsorption of Sodium Iodine at Air/Water Interface
}

\author{
Cuong V. Nguyen ${ }^{1}$, Hiromichi Nakahara ${ }^{2}$, Osamu Shibata ${ }^{3}$ and Chi M. Phan ${ }^{4, *}$ \\ ${ }^{1}$ Faculty of Applied Sciences, Ton Duc Thang University, Hochiminh City, 70000, Vietnam \\ ${ }^{2}$ Department of Industrial Pharmacy, Daiichi University of Pharmacy, 22-1 Tamagawa-cho, \\ Minami-ku Fukuoka 815-8511, Japan
} (1) Nagasaki International University, Sasebo, Nagasaki 859-3298, Japan.

${ }^{4}$ Discipline of Chemical Engineering and Curtin Institute of Functional Molecules and Interfaces, Curtin University, GPO Box U1987, Perth, WA 6845, Australia

*Corresponding author: c.phan@ curtin.edu.au

\section{ABSTRACT}

The change in surface potential was measured for NaI solutions. The modelled surface charge 5 was then calculated and compared with molecular simulations. It was found that $\mathrm{I}^{-}$was enhanced at the air/water interface more than $\mathrm{Na}^{+}$. The result, which was confirmed by simulations, was opposite to the previous observation with $\mathrm{NaCl}$. The trend is also consistent with anionic effects: larger and more polar anions adsorbed stronger at the air/water interface. The theoretical model was applied successfully to describe the changes for both systems, which are positive for $\mathrm{NaCl}$ and negative for $\mathrm{NaI}$, respectively. The combined results of the two systems also revealed that the self-ionization of pure water induced a positive surface charge at $16.9 \mathrm{mV}$.

KEYWORDS: sodium iodine, surface potential, surface charge 


\section{Introduction}

The adsorption of ions at the air/water interface is crucial to a proper understanding and explanation of various physical and chemical processes, such as mineral flotation [1] and atmospheric aerosols [2]. Thermodynamically, ions are expected to be repelled from the interface as the addition of salt to water triggers an increase in surface tension $[3,4]$. On the other hand, current studies of the reactivity of a variety of salt solutions have revealed that there exists an enhancement of anions at the liquid/vapour interface [5], which corresponds to the Hofmeister series [6]. Based on measurements of the uptake of gaseous $\mathrm{Br}_{2}$ and $\mathrm{Cl}_{2}$ by aqueous interfaces of sodium-halide salt solutions, $\mathrm{Hu}$ et al. [7] predicted that $\mathrm{Cl}^{-}$and $\mathrm{Br}^{-}$ions must exist at the air/water interface of those solutions. This prediction has been followed by a large number of studies employing molecular dynamics (MD) simulation. Instead of an ion-free interface, MD studies have demonstrated that some specific ions, such as $\mathrm{Cl}^{-}, \mathrm{Br}^{-}$and $\mathrm{I}^{-}$exhibit a propensity for the liquid/vapour interface [8-10]. In addition, other studies using state-of-theart techniques such as second-harmonic generation (SHG), vibrational sum-frequency generation (VSFG) and X-ray photoelectron spectroscopy (XPS) to validate the existence of ions near the surface [11-13]. It is noted that sodium-halide salts impact the structure of hydrogen bonding of water in the interfacial region at the level dependent on the nature of anions.

Combining experimental and computational results, our study has depicted the image of ionic distribution within the interfacial region of $\mathrm{NaCl}$ solutions [14]. More importantly, based on the newly proposed diffuse plane, the positively charged interface of $\mathrm{NaCl}$ solutions obtained by the measurement of their surface potentials are verified by the interaction between ions and interfacial water molecules acquired from MD simulations. In this study, we quantitatively evaluate the impact of anion nature on surface charge as well as the distribution of ions at the air/water interface by comparing the results of two salt solutions, $\mathrm{NaCl}$ and $\mathrm{NaI}$. 


\section{Theory}

50 As described in previous study and elsewhere [14,15], the alteration of surface potential of salt

51 solutions can be expressed as a function of surface charge:

$$
\Delta V=V-V_{0}=\frac{\lambda}{\varepsilon_{S} \varepsilon_{0}} \sigma+\operatorname{asinh}\left(\frac{\sigma}{\sqrt{8 C_{b} \varepsilon \varepsilon_{0} k_{B} T}}\right) \frac{2 k_{B} T}{e}
$$

53 where

$54 \quad V$ : change in surface potential $(\mathrm{V})$

55 : surface charge density $\left(\mathrm{C} / \mathrm{m}^{2}\right)$

$C_{b}:$ bulk particle concentration (particles $/ \mathrm{m}^{3}$ )

$57 \quad k_{B}$ : Boltzmann constant

$58 \quad T$ : temperature $(\mathrm{K})$

$59 e$ : charge of an electron

60 : permittivity of interfacial layer

$61 \quad{ }_{0}$ : vacuum permittivity

62 : permittivity of solution

$63 \lambda$ : the thickness of the interfacial layer

64 In the above equation, $V_{0}$ is surface potential of pure water. While $V_{0}$ is non-zero due to the 65 presence of hydronium/hydroxide ions, its actual value remains unqualified [16]. The solution 66 permittivity, $\varepsilon$, is dependent on the salinity [17]. The dependency is linear for $\mathrm{NaCl}$ and $\mathrm{NaI}$ up to $1.5 \mathrm{M}[18]$ and is given as:

$$
\varepsilon\left(C_{b}\right)=78.2-13.8 C_{b}
$$

69 The change in surface potential can be positive or negative depending on surface charge density of the interface, which is difficult to quantify in case of air/salt solution surface [19]. Previous investigation on the interface of $\mathrm{NaCl}$ solution employed $\mathrm{MD}$ simulation and directly quantified surface charge via the adsorption of ions:

$$
\sigma=e N_{a} \Gamma_{\text {ion }}=e N_{a}\left(\Gamma_{\text {cation }}-\Gamma_{\text {anion }}\right)
$$

74 where $N_{a}$ is Avogadro number, ion $\left(\mathrm{mol} / \mathrm{m}^{2}\right)$ is the net adsorption amount or concentration of 75 ions within the interfacial zone. 
76 Thermodynamically, the adsorption of ions within the interfacial zone is governed by their

77 interaction with surface water molecules, or the asymmetric H-bonds network [20]. The interaction would be influenced by the nature of cations and anions and determine the sign of surface charge density. In case of the $\mathrm{NaCl}$, the number of the adsorbed cations is greater than that of anion, resulting in positive surface charge density [14], which then was verified by

81 experimental surface potential.

\section{Computational and Experimental Methods}

83 Two empty regions representing vacuum (10 $\mathrm{nm}$ length each) were placed at both sides of a

84 slab of water layer (with a thickness of $10 \mathrm{~nm}$ ) to form two air/water interfaces. The simulation employed GROMACS 4.5.5 to generate the molecular trajectories with a time step of $1 \mathrm{fs}$.

Water model SPC/E was used and ions were described by OPLS force field [21,22]. Simulation boxes were formed following a widely accepted procedure. At first, a box $3 \times 3 \times 10$ of water molecules was built before replacing a number water molecule by $\mathrm{Na}^{+}$and $\mathrm{I}^{-}$ions for the purpose of increasing $\mathrm{NaI}$ concentration from 0 to $1.5 \mathrm{M}$ which is consistent with the experimental values. The box then was simulated at constant temperature ( $298 \mathrm{~K}$ and pressure (1 bar) employing Berendsen barostat with 2 ps relaxation time and $1.3 \mathrm{~nm}$ cut-off. Then, the $z$-dimension was extended to $30 \mathrm{~nm}$ to created two vacuum regions, while $x$-and $y$-dimension of the box were rescaled correspondingly. Finally, the simulation was run for $30 \mathrm{~ns}$ at constant volume and temperature (298 K) employing Nose-Hoover thermostat. Density distribution and water dipole moment were analysed based on the last $10 \mathrm{~ns}$ of simulation using GROMACS built-in functions. LINCS algorithms were used to keep the geometry of water molecules.

97 Electrostatic interactions were dealt with by employing Ewald sums.

In order to eliminate the broadening of the interface caused by capillary waves, the method of

99 identifying the truly interfacial molecules (ITIM) [23] was employed for analysing the last 10

100 ns of production simulation. The ITIM analyses have been conducted using PYTIM package

101 [24]. In this study, neighbouring test lines were separated by 0.4 A from each other based on 
102 the suggestions of previous studies [25-27] in literature. The radius of the probe sphere was

103 defined at 2.0 A, while the atoms' diameter was estimated by their Lennard-Jones distance

104 parameter. The procedure of ITIM analyses was repeated three times, and the molecules and

105 their number densities within the first three outer interfacial layers were determined.

106 The measurement of surface potential was conducted using an ionizing electrode as described

107 previously [28]. Sodium iodide (purity > 99.5\%) was obtained from Nacalai Tesque (Kyoto,

108 Japan) and was used as received. Each measurement was repeated at least 3 times to ensure its 109 repeatability.

\section{Results}

111 This study extended the previous definition of the interfacial zone to NaI solutions. Density

112 distribution of ions and water was obtained by using built-in function analysing the last $10 \mathrm{~ns}$

113 of each simulation. This length of analysing time was proved sufficient and reliable in the

114 previous study [14]. Instead of using Gibbs dividing plane, which is based on water density

115 profile, in this study surface limit is calculated from water dipole moment profile. Specifically,

116 the interfacial zone is defined corresponding to the peak of water dipole order as depicted in

117 Fig. 1.

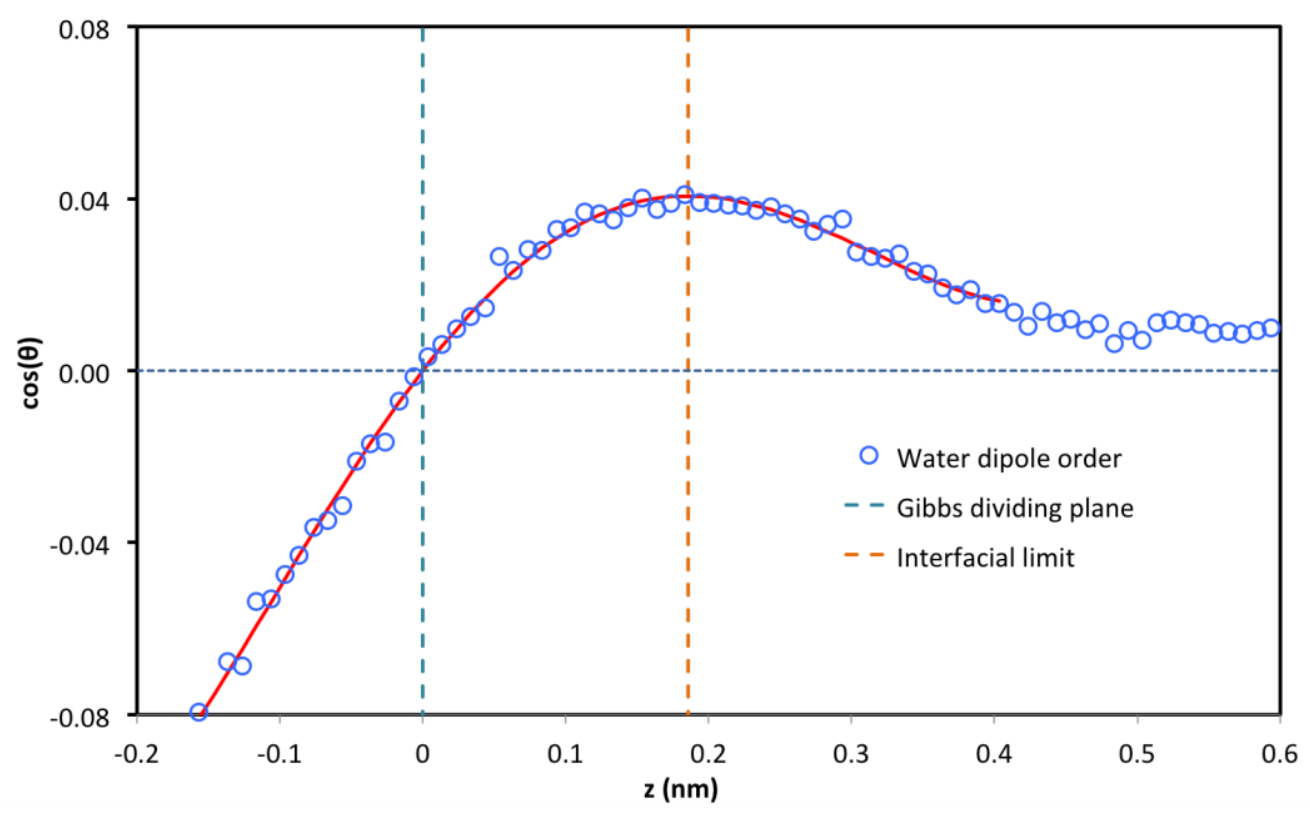


119 Fig. 1. Water distribution around the interfacial limit.

120 It should be noted that a newly-defined interfacial limited has been successfully applied to the 121 systems of alcohols aqueous solutions [29], alcohols/ $\mathrm{NaCl}$ mixture [30] and $\mathrm{NaCl}$ solution [14] 122 to quantify the molecular arrangement of the adsorption zone. Most importantly, this limiting 123 plane of interfacial zone helps verify the positive net ionic adsorption at the air/water interface 124 of $\mathrm{NaCl}$ solution, which is in contrast to traditional understanding of a negative adsorption at 125 air/liquid interface [15,31], where ions are expected to be depleted from the surface due to the 126 less polarization of outmost interfacial water layer [32]. The interfacial limit is employed to 127 describe the surface adsorption of NaI salt. Similarly, the net ionic adsorption is figured out 128 based on the accumulative number of ions as showed in Fig. 2.

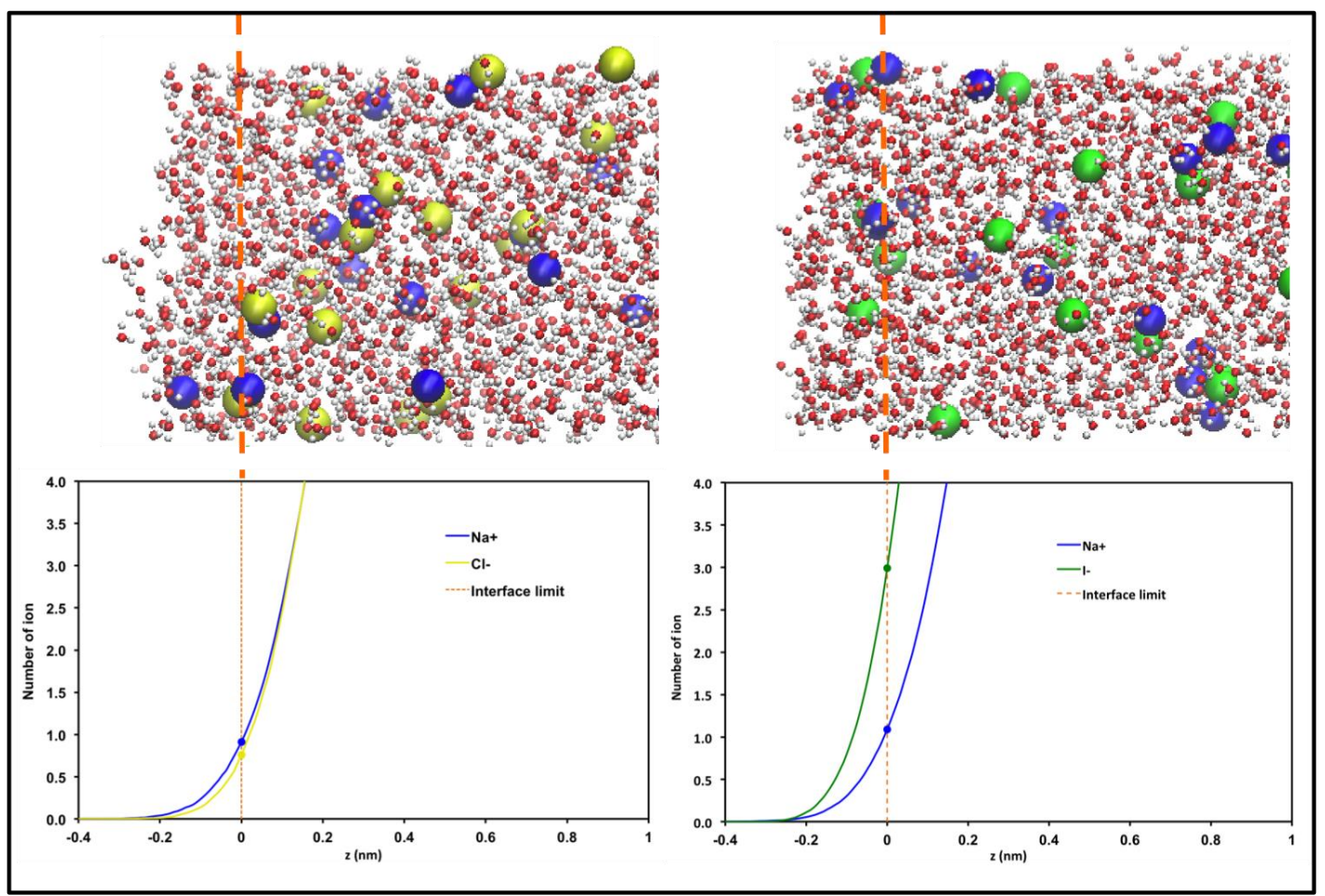

Fig. 2. Accumulative numbers of ions within the interfacial zone. $\mathrm{NaCl}$ (left) and $\mathrm{NaI}$ (right) 132 at the same concentration $(0.74 \mathrm{M})$

133 Ionic adsorption. There is a contradictive behaviour of ions within the interfacial zone between

134 the two systems. In both cases, the numbers of adsorbed $\mathrm{Na}^{+}$at the interface are relatively 135 similar. However, the relative anion/cation ratios are different. While the number of adsorbed $136 \mathrm{Cl}^{-}$is lower than that of $\mathrm{Na}^{+}$, there is significantly stronger adsorption of $\mathrm{I}^{-}$than $\mathrm{Na}^{+}$within this 
137 zone. This can be observed clearly on the snapshots of two simulations and result in the negative

138 net charge at the interface of NaI solution.

139 To exclude the capillary wave from the ionic distribution, number densities of $\mathrm{O}$ (water) across

140 the simulation box and the first 3 outmost layers (only the first layer was shown), ions was

141 determined following ITIM method and plotted in Fig. 3. Accumulated numbers of ions were

142 also figured out for the purpose of calculating the number of ions within the interfacial zone. It

143 is noted that in this instance, the limit of the interfacial layer was selected as the peak position

144 of the first layer of $\mathrm{O}$ (water) by selecting the maximum of the obtained Gaussian distribution

145 of the first layer. This limitation reasonably agreed with the position at which the mass water

146 density is $50 \%$ of the bulk density [23]. It can be seen in Fig. 3 that $\mathrm{I}^{-}$has higher concentration

147 than $\mathrm{Na}^{+}$. The data indicated that the relative difference between the ions is not affected by the 148 roughness of water surface.

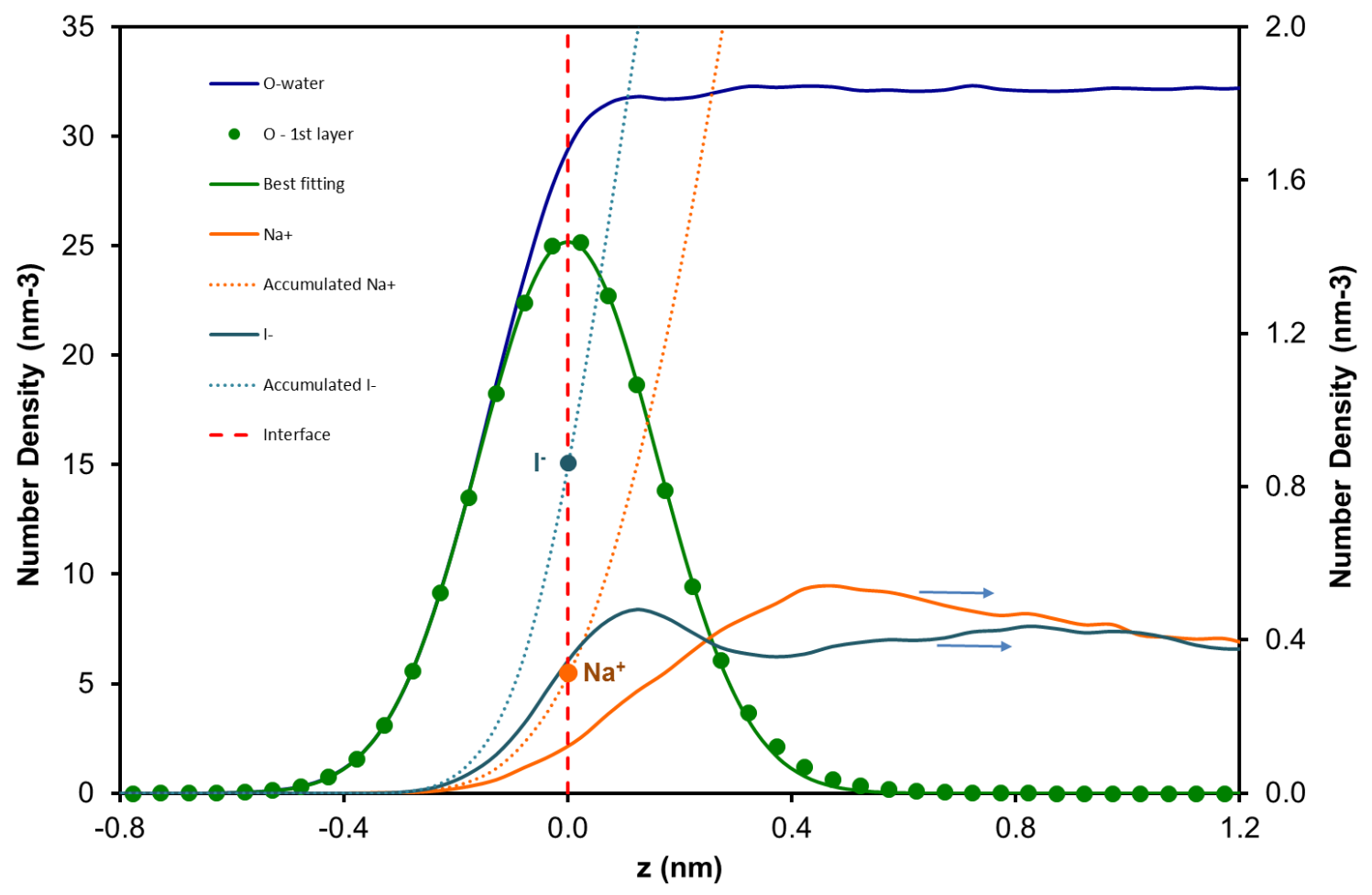

151 Fig. 3. Number of $\mathrm{O}$ (water) in the first layer and throughout the whole simulation box, ions and their accumulation within the interfacial zone $(0.74 \mathrm{M} \mathrm{NaI})$. 
154 The penetration of ions to the interface would be expectedly governed by the interaction with 155 surrounding water molecules. Thus, radial distributions functions (RDF) of water oxygen 156 molecules around ions were analysed and illustrated in Fig. 4.

157

158 Fig. 4. Radial distribution functions of water oxygen around ions in both $\mathrm{NaCl}$ and $\mathrm{NaI}$ 159 solutions.

160 Fig. 4 indicated a stable solvation shell around $\mathrm{Na}^{+}$ions in both solutions, which would be 161 responsible for almost similar adsorption of this cation at the interface regardless of different 162 companion anions. Nevertheless, the hydration layer of $\mathrm{I}^{-}$was less concentrated at a further 163 distance than that of $\mathrm{Cl}^{-}$. This would be driven by a larger ionic radii [33] of $\mathrm{I}^{-}$. Recently, it has 164 been shown the hydration shell of ions has a negative contribution to the surface tension by 165 interaction to surface water layer [34]. The observed trend follows the "hardness" order within 166 the Hofmeister series [3] and variation in $\mathrm{Na}^{+}$penetration depth with different anions [35]. 


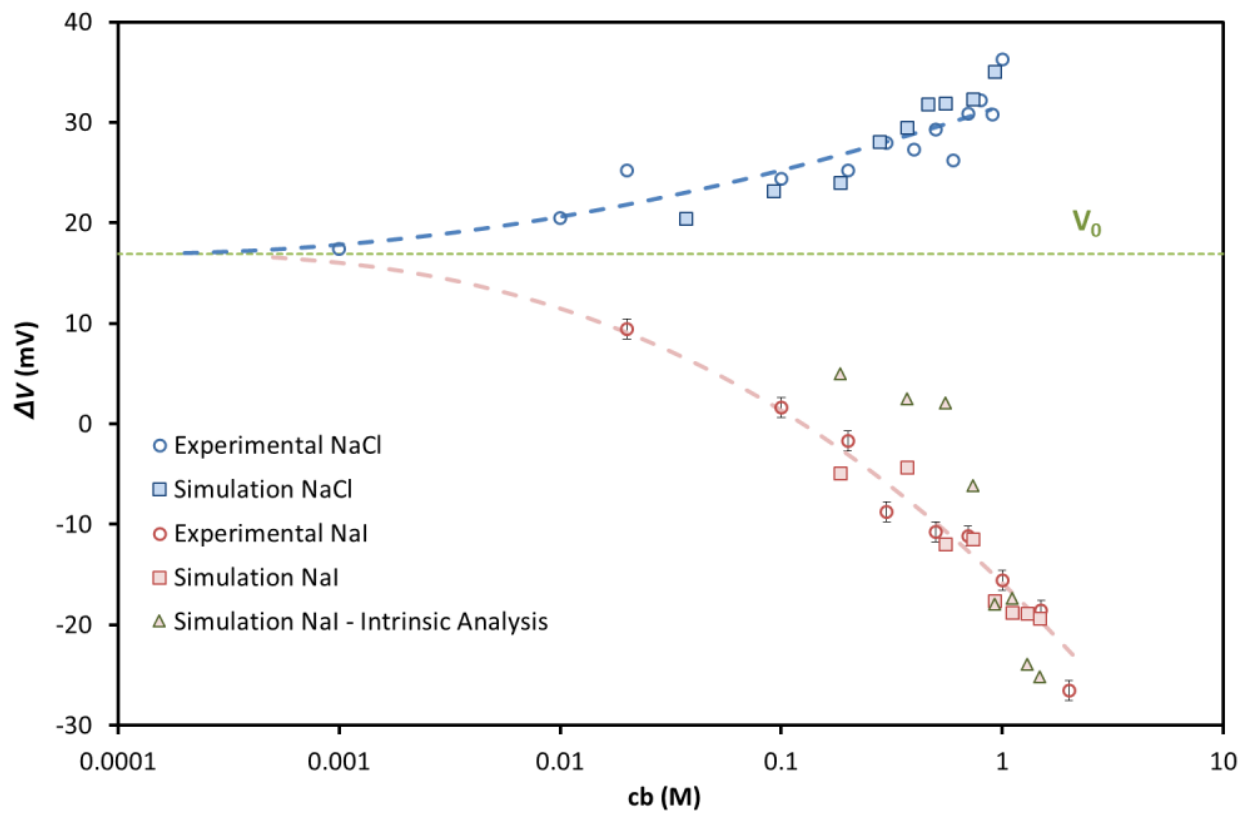

170

Fig. 5. Change in surface potential for $\mathrm{NaI}$ and $\mathrm{NaCl}$ solutions with an asymptotic limit at $C_{b}$ $\rightarrow 0$. The data for $\mathrm{NaCl}$ was taken from the previous study [14]. Lines are asymptotic curves. The best-fitted of $\lambda / \varepsilon \varepsilon_{\mathrm{s}}$ for NaI simulation was determined at 0.3 and $1.8 \mathrm{~F} / \mathrm{m}^{2}$, for $z$-axis distribution and ITIM analysis respectively.

Surface charge. The contrasting behaviour between $\mathrm{I}^{-}$and $\mathrm{Cl}^{-}$is also evident by the change in surface potential data (Fig. 5). In Eq.(1), the value of $\Delta V$ represents the re-organization of water dipole moment by salts. Hence, $\Delta V$ should approach zero as $C_{b} \rightarrow 0$. However, it can be seen in Fig.4b that $\Delta V$ does not approach zero as $C_{b}$ approach zero. By empirical fitting to both $\mathrm{NaCl}$ and $\mathrm{NaI}$ data, the asymptotic value was determined at $16.9 \mathrm{mV}$.

To corroborate the simulation with experimental data, the surface charge in Fig.4 was calculated by Eq. (1) and Eq. (2). The change in surface potential was adjusted by $16.9 \mathrm{mV}$ to account for the pure water surface. The surface charge was also obtained via simulation (Fig. 5). Both methods, density distribution and intrinsic analyses, demonstrated a negative surface charge, i.e. enhancement of $\mathrm{I}^{-}$over $\mathrm{Na}^{+}$. The charge is increased with increasing $\mathrm{NaI}$ concentration. The experimental and simulation data verified the contrasting behaviour between $\mathrm{NaCl}$ and $\mathrm{NaI}$. It is noteworthy that previous simulations showed similar effects of anions: $\mathrm{I}^{-}$is far more enhanced than $\mathrm{Cl}^{-}$near the surface [10]. However, the previous simulation showed that $\mathrm{Cl}^{-}$was also more enhanced than $\mathrm{Na}^{+}$, which is contrasting to our results [14]. 
189 Hence the simulated surface charge of $\mathrm{NaCl}$ solution is model-dependent. It should be

190 emphasized that the enhancement of $\mathrm{Na}^{+}$over $\mathrm{Cl}^{-}$was consistent with the experimental data,

191 which showed a positive charge. In both simulations, the relative difference $\mathrm{NaCl}$ is much small

192 than that of NaI, as demonstrated with Fig.6 and Fig.1 of Jungwirth and Tobias simulation [10].

193 In summary, the simulated ratio of $\mathrm{Na}^{+} / \mathrm{Cl}^{-}$is small and model-dependent. The simulated ratio

194 of $\mathrm{Na}^{+} / \mathrm{I}^{-}$is much larger, with a significant enhancement of $\mathrm{I}^{-}$, and consistent with the 195 experimental data.

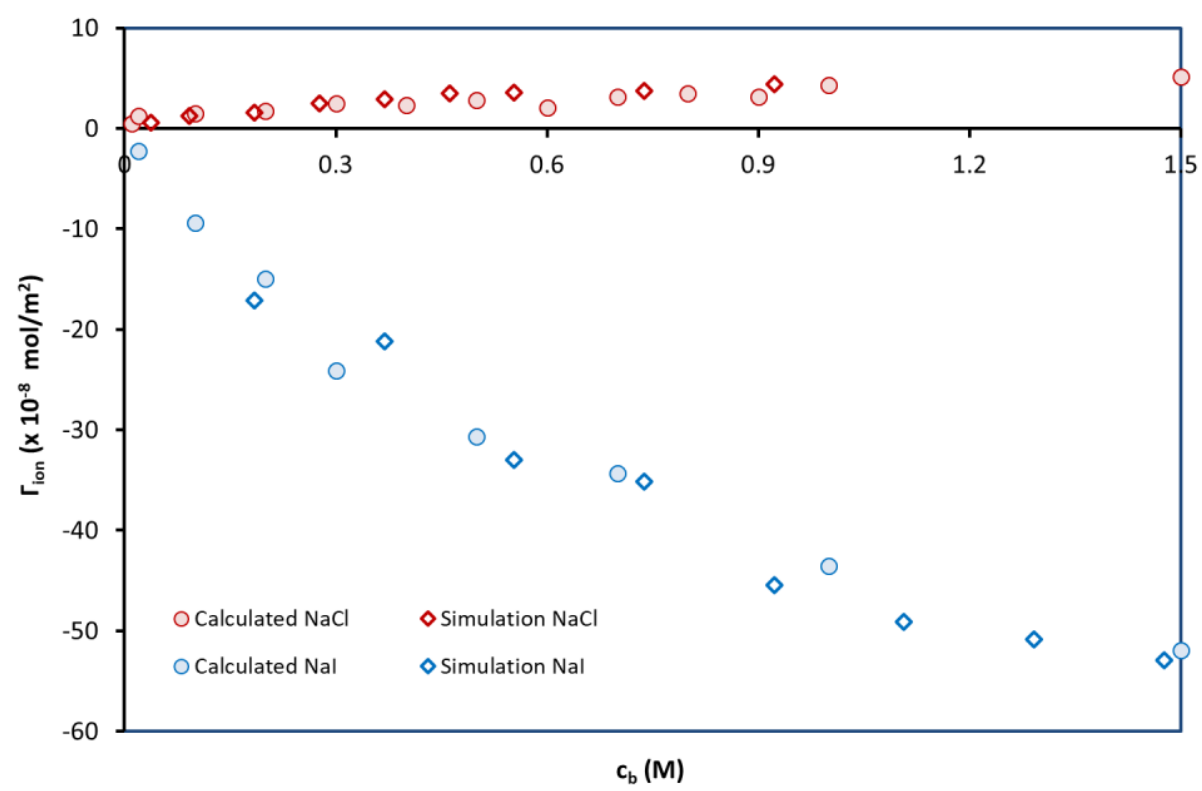

196

197

198

199

200

201

202

Fig. 6. Net ionic adsorption within the interfacial layer of $\mathrm{NaI}$ and $\mathrm{NaCl}$ solutions.

It can be seen from Fig. 6 that experimental and simulation data are consistent for both $\mathrm{NaI}$ and $\mathrm{NaCl}$. For the fitting, equation (3) has one parameter, $\lambda / \varepsilon \varepsilon_{\mathrm{s}}$, which incorporate the thickness and pemissivity of the interfacial layer. Since these properties cannot be quantified from simulation, the value are obtained by fitting against the experimental data.

The value of $\lambda / \varepsilon \varepsilon_{\mathrm{s}}$ for $\mathrm{NaI}$ (Fig. 5) was determined at $0.3 \mathrm{~F} / \mathrm{m}^{2}$, which is much smaller than that of $\mathrm{NaCl}$ [14]. This can be explained by the "harder" hydration shell of $\mathrm{I}^{-}$. As mentioned above, $\mathrm{I}^{-}$hydration shell has low polarization [9] and larger radii [33], which can increase $\lambda$ and 
decrease $\varepsilon_{s}$. The interaction of this hydration shell with surface water [34] can significantly

208 reduce the permittivity of the surface layer, $\varepsilon_{\mathrm{s}}$, as well.

209 Finally, it should be noted that the asymptotic value of surface potential is positive, which can

210 be attributed to the water orientation [36] or the relatively enhancement between hydronium/

211 hydroxide ions at the pure water surface [37]. However, the absolute value for ionic adsorption

212 for pure water is only obtainable from Eq.(1) if the value of $\lambda / \varepsilon \varepsilon_{\mathrm{s}}$ is known.

\section{Conclusions}

214 In summary, we investigated experimentally and theoretically the adsorption of $\mathrm{NaI}$ at the

215 air/water interface. It was consistently confirmed by both methods that $\mathrm{I}^{-}$has a higher adsorption

216 than $\mathrm{Na}^{+}$. The relative arrangement was opposite to the $\mathrm{NaCl}$ system, which can be attributed

217 to a larger hydration shell of $\mathrm{I}^{-}$. The results quantify the ionic effect, in this case halide ions, on

218 surface adsorption and surface potential. The results also validate the role of the water surface

219 structure in defining the limit of the interfacial layer. More interestingly, the study with these

220 electrolytes also indicates that the surface potential of pure water is positive, at $16.9 \mathrm{mV}$.

\section{AUTHOR INFORMATION}

222 The authors declare no competing financial interests.

\section{REFERENCES}

[1] C.V. Nguyen, C.M. Phan, H.M. Ang, H. Nakahara, O. Shibata, Y. Moroi, Surface potential of 1-hexanol solution: Comparison with methyl isobutyl carbinol, J. Phys. Chem. B. 117 (2013). doi:10.1021/jp4027157.

[2] V. Ramanathan, P.J. Crutzen, J.T. Kiehl, D. Rosenfeld, Aerosols, Climate, and the Hydrological Cycle, Sci. 294 (2001) 2119.

[3] L.M. Pegram, M.T. Record, Hofmeister Salt Effects on Surface Tension Arise from Partitioning of Anions and Cations between Bulk Water and the Air-Water Interface, J. Phys. Chem. B. 111 (2007) 5411-5417. doi:10.1021/jp070245z.

[4] P.B. Petersen, On the nature of ions at the liquid water surface, Annu. Rev. Phys. Chem. 57 (2006) 333.

[5] L. Sun, X. Li, Y. Tu, H. Agren, Origin of ion selectivity at the air/water interface, Phys. Chem. Chem. Phys. 17 (2015) 4311-4318. doi:10.1039/C4CP03338H.

[6] P. Jungwirth, P.S. Cremer, Beyond Hofmeister, Nat. Chem. 6 (2014) 261. doi:10.1038/nchem.1899. Uptake of $\mathrm{Cl} 2(\mathrm{~g})$ and $\mathrm{Br} 2(\mathrm{~g})$ by Aqueous Surfaces as a Function of Br- and I- Ion 
Concentration: The Effect of Chemical Reaction at the Interface, J. Phys. Chem. 99 (1995) 8768-8776. doi:10.1021/j100021a050.

[8] P. Jungwirth, D.J. Tobias, Specific ion effects at the air/water interface, Chem. Rev. 106 (2006) 1259-1281.

[9] P. Jungwirth, D.J. Tobias, Ions at the Air/Water Interface, J. Phys. Chem. B. 106 (2002) 6361-6373. doi:10.1021/jp020242g.

[10] P. Jungwirth, D.J. Tobias, Molecular Structure of Salt Solutions: A New View of the Interface with Implications for Heterogeneous Atmospheric Chemistry, J. Phys. Chem. B. 105 (2001) 10468-10472. doi:10.1021/jp012750g.

[11] E.A. Raymond, G.L. Richmond, Probing the Molecular Structure and Bonding of the Surface of Aqueous Salt Solutions, J. Phys. Chem. B. 108 (2004) 5051-5059. doi:10.1021/jp037725k.

[12] D. Liu, G. Ma, L.M. Levering, H.C. Allen, Vibrational Spectroscopy of Aqueous Sodium Halide Solutions and Air-Liquid Interfaces: Observation of Increased Interfacial Depth, J. Phys. Chem. B. 108 (2004) 2252-2260. doi:10.1021/jp036169r.

[13] S. Ghosal, J.C. Hemminger, H. Bluhm, M. Bongjin Simon, et al., Electron Spectroscopy of Aqueous Solution Interfaces Reveals Surface Enhancement of Halides, Science (80-. ). 307 (2005) 563-566.

[14] C. V. Nguyen, C.M. Phan, H. Nakahara, O. Shibata, Surface structure of sodium chloride solution, J. Mol. Liq. 248 (2017) 1039-1043. doi:10.1016/j.molliq.2017.10.138.

[15] H.-J. Butt, K. Graf, M. Kappl, Physics and chemistry of interfaces, John Wiley \& Sons, 2006.

[16] R. Zimmermann, U. Freudenberg, R. Schweiß, D. Küttner, C. Werner, Hydroxide and hydronium ion adsorption - A survey, Curr. Opin. Colloid Interface Sci. 15 (2010) 196-202. doi:10.1016/j.cocis.2010.01.002.

[17] C.T. Swift, An Improved Model for the Dielectric Constant of Sea Water at Microwave Frequencies, IEEE J. Ocean. Eng. 2 (1977) 104-111. doi:10.1109/JOE.1977.1145319.

[18] A. Levy, D. Andelman, H. Orland, Dielectric constant of ionic solutions: A field-theory approach, Phys. Rev. Lett. 108 (2012). doi:10.1103/PhysRevLett.108.227801.

[19] S.M. Kathmann, I.F.W. Kuo, C.J. Mundy, G.K. Schenter, Understanding the Surface Potential of Water, J. Phys. Chem. B. 115 (2011) 4369-4377. doi:10.1021/jp1116036.

[20] F. Perakis, L. De Marco, A. Shalit, F. Tang, Z.R. Kann, T.D. Kühne, R. Torre, M. Bonn, Y. Nagata, Vibrational Spectroscopy and Dynamics of Water, Chem. Rev. 116 (2016) 7590-7607. doi:10.1021/acs.chemrev.5b00640.

[21] W.L. Jorgensen, D.S. Maxwell, J. Tirado-Rives, Development and testing of the OPLS all-atom force field on conformational energetics and properties of organic liquids, J. Am. Chem. Soc. 118 (1996) 11225-11236.

[22] G.A. Kaminski, R.A. Friesner, J. Tirado-Rives, W.L. Jorgensen, Evaluation and Reparametrization of the OPLS-AA Force Field for Proteins via Comparison with Accurate Quantum Chemical Calculations on Peptides, J. Phys. Chem. B. 105 (2001) 6474-6487. doi:10.1021/jp003919d.

[23] L.B. Pártay, G. Hantal, P. Jedlovszky, Á. Vincze, G. Horvai, A new method for determining the interfacial molecules and characterizing the surface roughness in computer simulations. Application to the liquid-vapor interface of water, J. Comput. Chem. 29 (2008) 945-956. doi:10.1002/jcc.20852.

[24] M. Sega, G. Hantal, B. Fábián, P. Jedlovszky, Pytim: A python package for the interfacial analysis of molecular simulations, J. Comput. Chem. (2018). doi:10.1002/jcc.25384.

[25] M. Sega, The role of a small-scale cutoff in determining molecular layers at fluid interfaces, Phys. Chem. Chem. Phys. 18 (2016) 23354-23357. doi:10.1039/c6cp04788b. 
[26] M. Jorge, G. Hantal, P. Jedlovszky, M.N.D.S. Cordeiro, A critical assessment of methods for the intrinsic analysis of liquid interfaces: 2. Density profiles, J. Phys. Chem. C. 114 (2010) 18656-18663. doi:10.1021/jp107378s.

[27] R.A. Horváth, B. Fábián, M. Szőri, P. Jedlovszky, Investigation of the liquid-vapour interface of aqueous methylamine solutions by computer simulation methods, J. Mol. Liq. 288 (2019) 110978. doi:10.1016/J.MOLLIQ.2019.110978.

[28] C. V Nguyen, C.M. Phan, H.M. Ang, H. Nakahara, O. Shibata, Y. Moroi, Molecular Dynamics Investigation on Adsorption Layer of Alcohols at the Air/Brine Interface, Langmuir. 31 (2015) 50-56. doi:10.1021/la504471q.

[29] C.M. Phan, C. V Nguyen, T.T.T. Pham, Molecular Arrangement and Surface Tension of Alcohol Solutions, J. Phys. Chem. B. (2016). doi:10.1021/acs.jpcb.6b01209.

[30] G. Gao, C. V. Nguyen, C.M. Phan, Molecular arrangement between electrolyte and alcohol at the air/water interface, J. Mol. Liq. 242 (2017) 859-867. doi:10.1016/j.molliq.2017.07.083.

[31] O. Björneholm, M.H. Hansen, A. Hodgson, L.-M. Liu, D.T. Limmer, A. Michaelides, P. Pedevilla, J. Rossmeisl, H. Shen, G. Tocci, E. Tyrode, M.-M. Walz, J. Werner, H. Bluhm, Water at Interfaces, Chem. Rev. 116 (2016) 7698-7726. doi:10.1021/acs.chemrev.6b00045.

[32] L. Onsager, N.N.T. Samaras, The Surface Tension of Debye-Hückel Electrolytes, J. Chem. Phys. 2 (1934) 528-536. doi:10.1063/1.1749522.

[33] Y.Z. Wei, P. Chiang, S. Sridhar, Ion size effects on the dynamic and static dielectric properties of aqueous alkali solutions, J. Chem. Phys. 96 (1992) 4569-4573. doi:10.1063/1.462792.

[34] G. Hantal, M. Sega, G. Horvai, P. Jedlovszky, Contribution of Different Molecules and Moieties to the Surface Tension in Aqueous Surfactant Solutions, J. Phys. Chem. C. (2019) acs.jpcc.9b02553. doi:10.1021/acs.jpcc.9b02553.

[35] H. Tissot, G. Olivieri, J.J. Gallet, F. Bournel, M.G. Silly, F. Sirotti, F. Rochet, Cation Depth-Distribution at Alkali Halide Aqueous Solution Surfaces, J. Phys. Chem. C. 119 (2015) 9253-9259. doi:10.1021/jp512695c.

[36] Y.R. Shen, V. Ostroverkhov, Sum-frequency vibrational spectroscopy on water interfaces: Polar orientation of water molecules at interfaces, Chem. Rev. 106 (2006) 1140-1154. doi:10.1021/cr040377d.

[37] V. Buch, A. Milet, R. Vacha, P. Jungwirth, J.P. Devlin, Water surface is acidic, Proc. Natl. Acad. Sci. 104 (2007) 7342-7347. doi:10.1073/pnas.0611285104. 